\title{
Article
}

\section{CKM Gene rs8111989 Polymorphism and Power Athlete Status}

\author{
Valentina Ginevičienè ${ }^{1}$, Audronè Jakaitienè ${ }^{1}$, Algirdas Utkus ${ }^{1}$, Elliott C. R. Hall ${ }^{2}$, Ekaterina A. Semenova ${ }^{3,4}$, \\ Liliya B. Andryushchenko ${ }^{5}$, Andrey K. Larin ${ }^{3}$, Ethan Moreland ${ }^{2}{ }^{-}$, Edward V. Generozov ${ }^{3}$ and \\ Ildus I. Ahmetov $2,3,5,6, * \mathbb{D}$
}

check for updates

Citation: Ginevičienè, V.; Jakaitienè, A.; Utkus, A.; Hall, E.C.R.; Semenova, E.A.; Andryushchenko, L.B.; Larin, A.K.; Moreland, E.; Generozov, E.V.; Ahmetov, I.I. CKM Gene rs8111989 Polymorphism and Power Athlete Status. Genes 2021, 12, 1499. https://doi.org/10.3390/genes 12101499

Academic Editor: Juan Del Coso

Received: 21 August 2021

Accepted: 23 September 2021

Published: 25 September 2021

Publisher's Note: MDPI stays neutral with regard to jurisdictional claims in published maps and institutional affiliations.

Copyright: (c) 2021 by the authors. Licensee MDPI, Basel, Switzerland. This article is an open access article distributed under the terms and conditions of the Creative Commons Attribution (CC BY) license (https:/ / creativecommons.org/licenses/by/ $4.0 /)$.
1 Department of Human and Medical Genetics, Institute of Biomedical Science, Faculty of Medicine, Vilnius University, 01513 Vilnius, Lithuania; valentina.gineviciene@mf.vu.lt (V.G.); audrone.jakaitiene@mf.vu.lt (A.J.); algirdas.utkus@mf.vu.lt (A.U.)

2 Research Institute for Sport and Exercise Sciences, Liverpool John Moores University, Liverpool L3 5AF, UK; elliotthall@live.co.uk (E.C.R.H.); E.Moreland@2017.ljmu.ac.uk (E.M.)

3 Department of Molecular Biology and Genetics, Federal Research and Clinical Center of Physical-Chemical Medicine of Federal Medical Biological Agency, 119435 Moscow, Russia; alecsekaterina@gmail.com (E.A.S.); zelaz@yandex.ru (A.K.L.); generozov@gmail.com (E.V.G.)

4 Research Institute of Physical Culture and Sport, Volga Region State University of Physical Culture, Sport and Tourism, 420010 Kazan, Russia

5 Department of Physical Education, Plekhanov Russian University of Economics, 115093 Moscow, Russia; andryushenko-lil@mail.ru

6 Laboratory of Molecular Genetics, Kazan State Medical University, 420012 Kazan, Russia

* Correspondence: genoterra@mail.ru

\begin{abstract}
Multiple genetic variants are known to influence athletic performance. These include polymorphisms of the muscle-specific creatine kinase (CKM) gene, which have been associated with endurance and/or power phenotypes. However, independent replication is required to support those findings. The aim of the present study was to determine whether the CKM (rs8111989, c.*800A>G) polymorphism is associated with power athlete status in professional Russian and Lithuanian competitors. Genomic DNA was collected from 693 national and international standard athletes from Russia $(n=458)$ and Lithuania $(n=235)$, and 500 healthy non-athlete subjects from Russia $(n=291)$ and Lithuania $(n=209)$. Genotyping for the CKM rs8111989 (A/G) polymorphism was performed using PCR or micro-array analysis. Genotype and allele frequencies were compared between all athletes and non-athletes, and between non-athletes and athletes, segregated according to population and sporting discipline (from anaerobic-type events). No statistically significant differences in genotype or allele frequencies were observed between non-athletes and power athletes (strength-, sprint- and speed/strength-oriented) athletes. The present study reports the non-association of the CKM rs8111989 with elite status in athletes from sports in which anaerobic energy pathways determine success.
\end{abstract}

Keywords: anaerobic performance; creatine kinase; genetic variant; genotype

\section{Introduction}

Human physical performance is genetically influenced, particularly in sprint/power sports, with traits relating to muscular strength and power tending to have greater heritable components than endurance phenotypes [1]. Although the genetic contribution to athletic performance is undoubted, a conclusive list of the genetic variants involved, and the mechanisms underpinning their effect, remain unknown. Among several genes previously associated with athletic performance, the role of the muscle-specific creatine kinase $(C K M)$ in cellular energy homeostasis has led to the emergence of the CKM gene as an important candidate $[2,3]$.

Muscular energy provision is maintained by adenosine triphosphate (ATP) breakdown and the concomitant release of free energy. To sustain contractile activity, the rate of 
ATP regeneration must be complementary to ATP demand and is facilitated by the phosphagen, glycolytic, and mitochondrial respiration energy systems. Each system has specific substrates and byproducts, in addition to differences in maximal rates and capacities for ATP regeneration [4]. During short-duration, maximal-effort activities such as sprinting, strength training, and high-power sports, the circulatory system cannot fully supply the exercising musculature with the oxygen required for adequate mitochondrial respiration. Consequently, the phosphagen system utilizes intramuscular stores of high-energy phosphates, with creatine phosphate $(\mathrm{CrP})$ being the primary component of this system. The resynthesis of ATP from adenosine diphosphate (ADP) is catalyzed by the enzyme creatine kinase $(\mathrm{CrP}+\mathrm{ADP}+\mathrm{H}+\rightleftharpoons \mathrm{ATP}+\mathrm{Cr})$, meaning the efficiency of ATP generation is affected by the activity of creatine kinase $[4,5]$.

Creatine kinase (CK) can be comprised of the $\mathrm{M}$ (muscle) and $\mathrm{B}$ (brain) subunits, assembled into the homodimeric CK-M and CK-B enzymes or the CK-MB heterodimer. Each is expressed in a tissue-specific manner, with CK-M being the most abundant in skeletal muscle and CK-MB activity being the greatest in cardiac muscle. The CK-B homodimer is primarily expressed in nervous tissue, with small concentrations in skeletal muscle, whereas the additional CK isoforms Scmit-CK and Umit-CK are localized to the mitochondria. The abundance of CK-M in skeletal muscle means it is the most important $\mathrm{CK}$ isoform for energy regulation during exercise, with recent evidence suggesting that the CK-PCr system activity is genetically influenced [5]. The CK-M-encoding CKM gene is mapped to chromosome 19q13.2, and various studies have addressed the gene's role in skeletal muscle energy metabolism. A seminal study by van Deursen and colleagues showed that CK-M-knockout mice were unable to sustain maximal isometric contractions but had greater fatigue resistance and could perform a higher number of contractions to exhaustion [6]. Subsequent studies in rat models demonstrated that both the duration and intensity of endurance training have a negative linear relationship with CKM expression [7]. Additional animal studies focused on the consequences of CKM knockout in different muscle fiber types, reporting that $C K M$ activity is inversely correlated with aerobic capacity and endurance performance. Accordingly, targeted deletion using animal models has permitted the greater understanding of the role of CK-M in exercise performance, suggesting that higher expression may be detrimental to aerobic capacity but advantageous during anaerobic activity.

Human studies have focused on sequence variations in coding and noncoding regions of the CKM gene. More than 260 CKM single-nucleotide polymorphisms (SNPs) have been identified [8]. The most studied CKM SNP is rs8111989, where adenosine (A) is substituted with guanine $(\mathrm{G})$ at nucleotide position $800\left(\right.$ c. $\left.{ }^{*} 800 \mathrm{~A}>\mathrm{G}\right)$ [8]. Despite being located in the $3^{\prime}$ untranslated region and without altering the amino acid composition of CK-M, this variant purportedly affects CKM gene expression and CK-M activity [9]. Since the association of the rs8111989 A allele with endurance phenotypes before and after aerobic training was reported by Rivera and colleagues [10], several studies of the rs8111989 SNP have been published [3,5,11-15]. However, there is still a lack of consensus regarding the influence of the rs8111989 A and G alleles on athletic ability, meaning that replication studies using independent populations are needed to confirm or reject the association of rs8111989 with performance phenotypes. Therefore, the aim of this study was to determine whether the CKM (A/G) rs8111989 polymorphism is associated with elite power status in a cohort of Lithuanian and Russian athletes. We hypothesized that rs8111989 genotype distribution would differ between elite power athletes and non-athletes, and that the frequency of the G allele and GG genotype would be higher in athletes competing in sports that are reliant on anaerobic energy production.

\section{Materials and Methods}

\subsection{Subjects and Ethical Approval}

All procedures in this study conformed with ethical standards concerning the scientific research of sport and exercise, and were approved by the local Ethics Committees (Federal 
Research and Clinical Center of Physical-Chemical Medicine and the Lithuanian Bioethics Committee). Written informed consent was obtained from all participants and the study was conducted in compliance with the Declaration of Helsinki.

This case-control study included 458 Russian (RU) and 235 Lithuanian (LT) professional athletes ( 431 males and 262 females, aged $25.0 \pm 6.5$ years). All athletes were ranked in the top 10 nationally for their respective sporting disciplines and were prospectively stratified into three groups according to the duration and distance of their event, spanning a spectrum from sprint-oriented (athletes from strength/power sports with some aerobic component) to strength-oriented (athletes from strength/power sports with a predominantly anaerobic component). The sprint-oriented group (RU: $n=171$; LT: $n=83$ ) included athletes competing in short-distance races, including 100-400 m track-and-field sprinters, sprint cycling, rowing/canoe, and canoe-kayak sprints, $50-13100 \mathrm{~m}$ swim and 500-1000 $\mathrm{m}$ track races. The strength-oriented group (RU: $n=105 ; \mathrm{LT}: n=29)$ included weightlifters and powerlifters. The speed-/strength-oriented group (RU: $n=182$; LT: $n=123$ ) included athletes from track-and-field (throwing (push-throw), jump events (high jump, long jump, triple jump, and pole vault)), figure skating, alpine skiing, pentathlon, decathlon, heptathlon, rock climbing, rhythmic gymnastics, and wrestling. The inclusion criteria stipulated that athletes must have no history of positive tests for performanceenhancing substances under standard anti-doping controls. Non-athlete participants were 500 healthy, unrelated citizens ( 347 males and 153 females, aged $29.0 \pm 8.5$ years) from Russia $(n=291)$ and Lithuania $(n=209)$, all of whom had no competitive sporting experience. All participants were Caucasians.

\subsection{Genotyping}

For all Lithuanian samples, genomic DNA was extracted from peripheral blood leukocytes of the Lithuanian participants using a standard phenol-chloroform extraction method. Genotyping for CKM rs8111989 was performed in duplicate using an allelic discrimination assay on a 7900HT Fast real-time polymerase chain reaction (PCR) instrument (Applied Biosystems $^{\mathrm{TM}}$, Life Technologies, Waltham, MA, USA) with TaqMan probes (TaqMan ${ }^{\circledR}$ Pre-Designed SNP Genotyping Assay ID:C_3145002_10; Applied Biosystems ${ }^{\mathrm{TM}}$, Life Technologies, Waltham, MA, USA). Genotypes were assigned using software (SDS Software v2.3, Applied Biosystems ${ }^{\mathrm{TM}}$ ). In order to ensure proper internal control, positive and negative controls from different DNA aliquots were used for each genotype analysis.

For all Russian samples, molecular genetic analysis was performed with DNA samples obtained from leukocytes (venous blood). Four milliliters of venous blood was collected in tubes containing EDTA (Vacuette EDTA tubes; Greiner Bio-One, Kremsmünster, Austria). Blood samples were transported to the laboratory at $4{ }^{\circ} \mathrm{C}$, and DNA was extracted on the same day. DNA extraction and purification were performed using a commercial kit (Techno-Sorb, Moscow, Russia) according to the manufacturer's instructions (Technoclon, Moscow, Russia), which included chemical lysis, selective DNA binding on silica spin columns, and ethanol washing. The extracted DNA quality was assessed by means of agarose gel electrophoresis. The genotyping process was performed using HumanOmni1Quad BeadChips or HumanOmniExpress BeadChips (Illumina, San Diego, CA, USA) to genotype $>900,000$ SNPs, including CKM rs8111989. The assay required $200 \mathrm{ng}$ of DNA sample as input with a concentration of at least $50 \mathrm{ng} / \mu \mathrm{L}$. Exact concentrations of DNA in each sample were measured using a Qubit Fluorometer (Invitrogen, Waltham, MA, USA). All further procedures were performed according to the instructions of the Infinium High-Density Assay. Ten percent of samples were genotyped twice with a $100 \%$ success rate of reproducibility.

\subsection{Statistical Analyses}

Genotype frequencies of athletes and non-athletes were tested for compatibility with the Hardy-Weinberg equilibrium (HWE). Differences in genotype and allele frequency were compared using the Chi-squared goodness-of-fit test. The homogeneity hypothesis for 
genotype and allele frequency differences between groups were assessed by Chi-squared or Fisher's exact tests where appropriate. Odds ratios (OR) with $95 \%$ confidence intervals (CIs) were calculated in cases where there was a significant difference in the genotype distribution between groups. The level of significance was set at $p<0.05$. All analysis was performed using the SPSS statistical software package (IBM SPSS v.21).

\section{Results}

The distributions of CKM rs8111989 genotypes in athletes versus controls are presented in Table 1. Genotype distributions were in the Hardy-Weinberg Equilibrium for athletes and non-athletes (in all groups tested separately, $p>0.05$ ).

Table 1. Genotype and allele frequencies of the CKM rs8111989 SNP in athletes and non-athletes from Russia and Lithuania.

\begin{tabular}{|c|c|c|c|c|c|c|c|c|}
\hline \multirow{2}{*}{ Groups } & \multirow{2}{*}{$\mathbf{N}$} & \multicolumn{2}{|c|}{ Allele Frequency (\%) } & \multirow{2}{*}{$\begin{array}{c}p \text {-Value vs. } \\
\text { Non-Athletes }\end{array}$} & \multicolumn{3}{|c|}{ CKM Genotype Frequency (\%) } & \multirow{2}{*}{$\begin{array}{c}p \text {-Value vs. } \\
\text { Non-Athletes }\end{array}$} \\
\hline & & A & G & & AA & AG & GG & \\
\hline All strength-oriented & 134 & 67.2 & 32.8 & 0.496 & $65(48.5)$ & $50(37.3)$ & $19(14.2)$ & 0.246 \\
\hline All sprint-oriented & 254 & 64.4 & 35.6 & 0.914 & $107(42.1)$ & $113(44.5)$ & $34(13.4)$ & 0.980 \\
\hline All speed/strength & 305 & 65.2 & 34.8 & 0.720 & $136(44.6)$ & $126(41.3)$ & $43(14.1)$ & 0.556 \\
\hline Russian power athletes & 458 & 65.6 & 34.4 & 0.988 & $204(44.5)$ & $193(42.2)$ & $61(13.3)$ & 0.915 \\
\hline Lithuanian power athletes & 235 & 64.7 & 35.3 & 0.858 & $104(44.3)$ & $96(40.8)$ & $35(14.9)$ & 0.534 \\
\hline All power athletes & 693 & 65.3 & 34.7 & 0.98 & $308(44.4)$ & $289(41.7)$ & $96(13.9)$ & 0.475 \\
\hline Russian non-athletes & 291 & 66.7 & 33.3 & - & $133(45.7)$ & $122(41.9)$ & $36(12.4)$ & - \\
\hline Lithuanian non-athletes & 209 & 60.3 & 39.7 & - & $74(35.4)$ & $104(49.8)$ & $31(14.8)$ & - \\
\hline All non-athletes & 500 & 64.0 & 36.0 & - & $207(41.4)$ & $226(45.2)$ & $67(13.4)$ & - \\
\hline
\end{tabular}

No statistically significant differences in CKM genotype or allele distribution were observed compared to non-athletes when all athletes were combined, nor when athletes were segregated by country (Table 1). In addition, CKM genotype and allele frequencies did not differ between male and female athletes and non-athletes for each nationality.

\section{Discussion}

Sports events that involve short intense efforts are often categorized as anaerobic athletic activities. Each of these events may emphasize different anaerobic phenotypes, for example, sprinting relies mostly on speed, weightlifting or powerlifting rely mainly on muscle strength, and throwing and jump events rely on the combination of speed and strength [15-17]. Therefore, the aim of this study was to investigate differences in the CKM rs8111989 genotype between three groups of elite athletes from anaerobic-type events: sprint-oriented, strength-oriented, and speed/strength-oriented athletes. We chose to study the rs8111989 genetic variant because the CKM gene has emerged as a potential candidate to affect the development of anaerobic phenotypes. We hypothesized that a genetic predisposition for high CK-M protein activity in skeletal muscle may be favorable to power/strength athletes, and specifically that the GG genotype would be more common in strength and power competitors, due to previous associations with increased CK-M activity. This theory is supported by rodent models documenting the inverse relationship of CKM activity with endurance capacity [7], suggesting that high CKM activity may impair aerobic performance yet favor anaerobic activity. This study led us to conclude that the genotype and/or allele frequencies of the CKM rs 8111989 polymorphism do not seem to influence performance in either the total strength/power group or in sports groups from two discrete populations. Nevertheless, this study emphasizes the importance of considering well-defined groups of athletes with a clear phenotype in genetic association studies applied to elite sport performance.

Muscular strength is a determinant of general athletic ability in many sports, particularly in short-distance sprinting, and strength and power events, in which the physiological characteristics favorable to national and international standard competition are well documented $[12,15,16,18]$. In contrast with endurance competitors, strength and power athletes 
typically exhibit greater percentages of fast-twitch muscle fibers than slow-twitch fibers [17], and those fast-twitch fibers are suited to the shorter, more powerful muscle contractions reliant on anaerobic energy pathways. The muscle-specific CK-M enzyme catalyzes the reversible transfer of energy-rich phosphate from $\mathrm{CrP}$ to $\mathrm{ADP}$ and is essential for energy provision during contractile activity, with higher CK-M activity in fast-twitch compared to slow-twitch fibers $[4,5]$. Consequently, the CKM gene has emerged as a candidate that might affect the development of aerobic and/or anaerobic phenotypes. Furthermore, according to the GTEx portal, the G allele of the rs8111989 polymorphism is associated with decreased expression of the MARK4 gene in skeletal muscle, which acts as a negative regulator of the mTORC1 complex (a key factor in maintaining skeletal muscle mass) [19]. Therefore, we hypothesized that a genetic predisposition for high CK-M protein activity may be advantageous to athletes in sports that are reliant on the contractile capacity of fast-twitch muscle fibers, and specifically that variants associated with increased CK-M and mTORC1 activity would be more common in strength competitors. The A-to-G substitution of the rs8111989 SNP does not change the CK-M amino acid sequence but reportedly alters CKM mRNA stability [12] and potentially CK-M expression. Further studies are required to determine whether this mechanism influences CKM expression and skeletal muscle phenotypes.

Our findings are not supported by a recent meta-analysis of CKM rs8111989 genotypes and/or allele distribution in athletes and non-athletes, where the frequencies of the $G$ allele and GG genotype were higher in power athletes than in non-athletes [8]. The rs8111989 SNP may also contribute to baseline aerobic capacity and differences in the response of maximal oxygen uptake to endurance training $[10,11,20,21]$. Fedotovskaya and colleagues reported that the AA genotype is associated with high values of maximal oxygen uptake [14], whereas the $G$ allele has been associated with combat athlete status in Polish and Russian populations [22]. Although also reliant on fast-twitch muscle fibers, sprint- and speed/strength-oriented sports involve a greater aerobic contribution than weightlifting and powerlifting, potentially explaining why the GG genotype was not overrepresented in these cohorts. Athletes with the rs8111989 AA genotype are also six times more likely to suffer exertional muscle breakdown than AG or GG genotypes [11,23], leading to the suggestion that the $G$ allele protects against exertional muscle damage $[13,24]$. Since fasttwitch fibers are more susceptible to exertional muscle damage [24], genetic resistance to exertional muscle damage would be advantageous to strength-oriented athletes who perform repetitive maximal contractions. However, the findings of the present study do not indicate that the rs $8111989 \mathrm{G}$ allele carriers are predisposed to excel in strength and power sports.

A growing body of evidence supports an association of the rs8111989 AA and GG genotypes with endurance and strength phenotypes, respectively $[12,17,21,22,25-28]$. However, a tendency for $G$ allele carriers to achieve higher maximum oxygen uptake values has been reported [27], and several studies (together with the present study) have failed to detect any association with athlete status or performance $[8-10,13,23]$. This demonstrates that further investigation surrounding CKM variants and athletic performance phenotypes is required. To date, 62 genetic markers have been associated with strength-power athlete status [3], but only some were reported in strength sports groups (weightlifting and powerlifting). These include SNPs of CNTFR, ACTN3, AGT, AR, HIF1A, PPARA, PPARG, PPARGC1A, and other genes [3,9,17,29-36]. Recently, Grishina and colleagues confirmed the association of three SNPs (rs12055409, rs4626333, and rs2273555) with strength measures in weightlifters, which were supported by functional evidence [31,32], whereas the most comprehensive study of elite strength athletes (weightlifters and powerlifters) to date documented 28 SNPs associated with athlete status [33]. Of those 28 SNPs, the LRPPRC rs10186876, MMS22L rs9320823, and PHACTR1 rs6905419 polymorphisms were also associated with competitive weightlifting performance. Importantly, that study was the first to demonstrate the likelihood that achieving elite status in strength sports depends on a high number of SNPs [33]. Therefore, further studies are needed to replicate those findings 
and to confirm whether those SNPs are associated with performance in strength-oriented sports. This study does not confirm the previous associations of the CKM rs8111989 GG genotype with power athlete status [22]. Additional studies of elite power athletes from different nationalities are required to further substantiate our findings.

We acknowledge the limitations of the present study. Firstly, we did not include endurance athletes and are unable to ascertain whether the rs8111989 A and G alleles are associated with contrasting performance phenotypes. Second, participant nationalities were restricted to Russia and Lithuania, meaning that the associations described in the present study cannot be generalized to athletes from other countries or geographical ancestries. We also did not collect direct measures of athletes' strength and power. However, our inclusion criteria stipulated that all athletes had competed at the national or international level, which would not be possible without the physiological characteristics associated with elite strength phenotypes. Lastly, we studied a singular SNP and recognize that genetic association studies represent only the first steps toward understanding the genetic factors influencing performance traits. However, candidate gene association studies are crucial to the direction of new analyses, such as those incorporating modern DNA technologies and bioinformatics, to further analyze the heritability of physical capacity and to work toward the application of genomic research into practice.

\section{Conclusions}

The present study reports the non-association of the CKM rs8111989 polymorphism with elite status in athletes from sports in which anaerobic energy pathways determine success. Therefore, the CKM rs8111989 polymorphism may not be considered as a candidate gene to influence the performance of power athletes from Russia and Lithuania.

Author Contributions: Conceptualization, V.G., A.U. and I.I.A.; methodology, V.G. and I.I.A.; software, V.G. and I.I.A.; validation, V.G., A.J. and I.I.A.; formal analysis, V.G.; investigation, V.G., E.C.R.H., E.A.S., L.B.A., A.K.L., E.M., E.V.G. and I.I.A.; resources, V.G. and A.U.; data curation, A.J.; writing—original draft preparation, V.G.; writing—review and editing, A.U., E.C.R.H., E.M. and I.I.A. All authors have read and agreed to the published version of the manuscript.

Funding: This research received no external funding.

Institutional Review Board Statement: The study was conducted according to the guidelines of the Declaration of Helsinki and approved by the Lithuanian Bioethics Committee (protocol code 69-99-111) and the Ethics Committee of the Federal Research and Clinical Center of PhysicalChemical Medicine.

Informed Consent Statement: Informed consent was obtained from all subjects involved in the study.

Data Availability Statement: The data presented in this study are available on request from the corresponding author.

Conflicts of Interest: The authors declare no conflict of interest.

\section{References}

1. Zempo, H.; Miyamoto-Mikami, E.; Kikuchi, N.; Fuku, N.; Miyachi, M.; Murakami, H. Heritability estimates of muscle strengthrelated phenotypes: A systematic review and meta-analysis. Scand. J. Med. Sci. Sports 2017, 27, 1537-1546. [CrossRef]

2. Maciejewska-Skrendo, A.; Sawczuk, M.; Cięszczyk, P.; Ahmetov, I.I. Genes and Power Athlete Status. In Sports, Exercise, and Nutritional Genomics: Current Status and Future Directions; Barh, D., Ahmetov, I., Eds.; Academic Press: Cambridge, MA, USA, 2019; pp. 41-72.

3. Ahmetov, I.I.; Hall, E.C.R.; Semenova, E.A.; Pranckevičienė, E.; Ginevičienè, V. Advances in sports genomics. Adv. Clin. Chem. 2021. [CrossRef]

4. Baker, J.S.; McCormick, M.C.; Robergs, R.A. Interaction among Skeletal Muscle Metabolic Energy Systems during Intense Exercise. J. Nutr. Metab. 2010, 2010, 905612. [CrossRef]

5. Echegaray, M.; Rivera, M.A. Role of creatine kinase isoenzymes on muscular and cardiorespiratory endurance: Genetic and molecular evidence. Sports Med. 2001, 31, 919-934. [CrossRef]

6. van Deursen, J.; Heerschap, A.; Oerlemans, F.; Rultenbeek, W.; Jap, P.; ter Laak, H.; Wieringa, B. Skeletal muscle of mice deficient in muscle creatine kinase lack burst activity. Cell 1993, 74, 621-631. [CrossRef] 
7. Apple, F.S.; Billardello, J.J. Expression of creatine kinase M and B mRNAs in treadmill trained rat skeletal muscle. Life Sci. 1994, 55, 585-592. [CrossRef]

8. Chen, C.; Sun, Y.; Liang, H.; Yu, D.; Hu, S. A meta-analysis of the association of CKM gene rs8111989 polymorphism with sport performance. Biol. Sport 2017, 34, 323-330. [CrossRef] [PubMed]

9. Grealy, R.; Herruer, J.; Smith, C.L.E.; Hiller, D.; Haseler, L.J.; Griffiths, L.R. Evaluation of a 7-Gene Genetic Profile for Athletic Endurance Phenotype in Ironman Championship Triathletes. PLoS ONE 2015, 10, e0145171.

10. Rivera, M.A.; Dionne, F.T.; Wolfarth, B.; Chagnon, M.; Simoneau, J.A.; Perusse, L.; Boulay, M.R.; Gagnon, J.; Song, T.M.; Keul, J.; et al. Muscle-specific creatine kinase gene polymorphisms in elite endurance athletes and sedentary controls. Med. Sci. Sports Exerc. 1997, 29, 1444-1447. [CrossRef] [PubMed]

11. Baird, M.F.; Graham, S.M.; Baker, J.S.; Bickerstaff, G.F. Creatine-kinase- and exercise-related muscle damage implications for muscle performance and recovery. J. Nutr. Metab. 2012, 2012, 960363. [CrossRef]

12. Batavani, M.R.; Marandi, S.M.; Ghaedi, K.; Esfarjani, F. Comparison of Muscle-Specific Creatine Kinase (CK-MM) Gene Polymorphism (rs8111989) Among Professional, Amateur Athletes and Non-athlete Karatekas. Asian J. Sports Med. 2017, 8, e43210. [CrossRef]

13. Eider, J.; Ahmetov, I.I.; Fedotovskaya, O.N.; Moska, W.; Cieszczyk, P.; Zarebska, A.; Czubek, Z.; Klocek, T.; Stepien-Slodkowska, M.; Maciejewska-Karlowska, A.; et al. CKM gene polymorphism in Russian and Polish rowers. Genetika 2015, 51, 389-392. [CrossRef]

14. Fedotovskaya, O.N.; Popov, D.V.; Vinogradova, O.L.; Akhmetov, I.I. Association of the muscle-specific creatine kinase (CKMM) gene polymorphism with physical performance of athletes. Fiziol. Cheloveka 2012, 38, 105-109. [CrossRef]

15. Latella, C.; Van den Hoek, D.; Teo, W.-P. Factors affecting powerlifting performance: An analysis of age- and weight-based determinants of relative strength. Int. J. Perform. Anal. Sport 2018, 18, 532-544. [CrossRef]

16. Aasa, U.; Svartholm, I.; Andersson, F.; Berglund, L. Injuries among weightlifters and powerlifters: A systematic review. Br. J. Sports Med. 2017, 51, 211-220. [CrossRef] [PubMed]

17. Ben-Zaken, S.; Eliakim, A.; Nemet, D.; Meckel, Y. Genetic variability among power athletes: The stronger vs. the faster. J. Strength Cond. Res. 2019, 33, 1505-1511. [CrossRef] [PubMed]

18. Tikkanen, E.; Gustafsson, S.; Amar, D.; Shcherbina, A.; Waggott, D.; Ashley, E.A.; Ingelsson, E. Biological Insights into Muscular Strength: Genetic Findings in the UK Biobank. Sci. Rep. 2018, 8, 6451. [CrossRef]

19. Li, L.; Guan, K.L. Microtubule-associated protein/microtubule affinity-regulating kinase 4 (MARK4) is a negative regulator of the mammalian target of rapamycin complex 1 (mTORC1). J. Biol. Chem. 2013, 288, 703-708. [CrossRef]

20. Zhou, D.Q.; Hu, Y.; Liu, G.; Gong, L.; Xi, Y.; Wen, L. Muscle-specific creatine kinase gene polymorphism and running economy responses to an 18-week 5000-m training programme. Br. J. Sports Med. 2006, 40, 988-991. [CrossRef]

21. Martinez, J.L.; Khorsandi, S.; Sojo, R.; Martínez, C.; Martín, J.A.; López-Taylor, J.R.; Fahey, T.D.; Rivera, M.A. Lack of an association between CKMM genotype and endurance performance level in Hispanic marathon runners. Med. Sport 2009, 13, 219-223. [CrossRef]

22. Fedotovskaya, O.; Eider, J.; Cięszczyk, P.; Ahmetov, I.; Moska, W.; Sawczyn, S.; Leońska-Duniec, A.; Maciejewska-Karłowska, A.; Sawczuk, M.; Czubek, Z.; et al. Association of muscle-specific creatine kinase (CKM) gene polymorphism with combat athlete status in Polish and Russian cohorts. Arch. Budo 2013, 9, 33-237.

23. Heled, Y.; Bloom, M.S.; Wu, T.J.; Stephens, Q.; Deuster, P.A. CKMM and ACE genotypes and physiological prediction of the creatine kinase response to exercise. J. Appl. Physiol. 2007, 103, 504-510. [CrossRef]

24. Macaluso, F.; Isaacs, A.W.; Myburgh, K.H. Preferential type II muscle fiber damage from plyometric exercise. J. Athl. Train. 2012, 47, 414-420. [CrossRef]

25. Ruiz, J.R.; Gomez-Gallego, F.; Santiago, C.; González-Freire, M.; Verde, Z.; Foster, C.; Lucia, A. Is there an optimum endurance polygenic profile? J. Physiol. 2009, 587 Pt 7, 1527-1534. [CrossRef] [PubMed]

26. Sprouse, C.; Tosi, L.L.; Gordish-Dressman, H.; Abdel-Ghani, M.S.; Panchapakesan, K.; Niederberger, B.; Devaney, J.M.; Kelly, K.R. CK-MM Polymorphism is Associated with Physical Fitness Test Scores in Military Recruits. Mil. Med. 2015, 180, 1001-1005. [PubMed]

27. Gronek, P.; Holdys, J.; Krysciak, J.; Stanislawski, D. CKM Gene G (Ncoi-) Allele Has a Positive Effect on Maximal Oxygen Uptake in Caucasian Women Practicing Sports Requiring Aerobic and Anaerobic Exercise Metabolism. J. Hum. Kinet 2013, 39, 137-145. [CrossRef]

28. He, E.; Li, Y.; Qian, J.; Yan, H. Association of CKMM gene A/G polymorphism and athletic performance of Uyghur nationality. Zhongguo Ying Yong Sheng Li Xue Za Zhi 2016, 32, 82-86.

29. Ahmetov, I.I.; Mozhayskaya, I.A.; Lyubaeva, E.V.; Vinogradova, O.L.; Rogozkin, V.A. PPARG Gene polymorphism and locomotor activity in humans. Bull. Exp. Biol. Med. 2008, 146, 630-632. [CrossRef]

30. Gabbasov, R.T.; Arkhipova, A.A.; Borisova, A.V.; Hakimullina, A.M.; Kuznetsova, A.V.; Williams, A.G.; Day, S.H.; Ahmetov, I.I. The HIF1A gene Pro582Ser polymorphism in Russian strength athletes. J. Strength Cond. Res. 2013, 27, 2055-2058. [CrossRef]

31. Grishina, E.E.; Zmijewski, P.; Semenova, E.A.; Cieszczyk, P.; Huminska-Lisowska, K.; Michałowska-Sawczyn, M.; Maculewicz, E.; Crewther, B.; Orysiak, J.; Kostryukova, E.S.; et al. Three DNA polymorphisms previously identified as markers for handgrip strength are associated with strength in weightlifters and muscle fiber hypertrophy. J. Strength Cond. Res. 2019, 33, $2602-2607$. [CrossRef] 
32. Homma, H.; Kobatake, N.; Sekimoto, Y.; Saito, M.; Mochizuki, Y.; Okamoto, T.; Nakazato, K.; Nishiyama, T.; Kikuchi, N. Ciliary Neurotrophic Factor Receptor rs41274853 Polymorphism is Associated with Weightlifting Performance in Japanese Weightlifters. J. Strength Cond. Res. 2020, 34, 3037-3041. [CrossRef] [PubMed]

33. Moreland, E.; Borisov, O.V.; Semenova, E.A.; Larin, A.K.; Andryushchenko, O.N.; Andryushchenko, L.B.; Generozov, E.V.; Williams, A.G.; Ahmetov, I.I. Polygenic profile of elite strength athletes. J. Strength Cond. Res. 2020. [CrossRef] [PubMed]

34. Gineviciene, V.; Jakaitiene, A.; Aksenov, M.O.; Aksenova, A.V.; Druzhevskaya, A.M.; Astratenkova, I.V.; Egorova, E.S.; Gabdrakhmanova, L.J.; Tubelis, L.; Kucinskas, V.; et al. Association analysis of ACE, ACTN3 and PPARGC1A gene polymorphisms in two cohorts of European strength and power athletes. Biol. Sport 2016, 33, 199-206. [CrossRef]

35. Zarebska, A.; Ahmetov, I.I.; Sawczyn, S.; Weiner, A.S.; Kaczmarczyk, M.; Ficek, K.; Maciejewska-Karlowska, A.; Sawczuk, M.; Leonska-Duniec, A.; Klocek, T.; et al. Association of the MTHFR 1298A>C (rs1801131) polymorphism with speed and strength sports in Russian and Polish athletes. J. Sports Sci. 2014, 32, 375-382. [CrossRef] [PubMed]

36. Guilherme, J.P.L.F.; Shikhova, Y.V.; Dondukovskaya, R.R.; Topanova, A.A.; Semenova, E.A.; Astratenkova, I.V.; Ahmetov, I.I. Androgen receptor gene microsatellite polymorphism is associated with muscle mass and strength in bodybuilders and power athlete status. Ann. Hum. Biol. 2021, 48, 142-149. [CrossRef] [PubMed] 